\title{
Optimal Designs for the Multiresponse Polynomial Regression Models in Symmetric Domains
}

\author{
Xuanhao Zhang \\ College of Science, Shanghai Second Polytechnic University, Shanghai, 201209, China \\ E-mail: xhzhang@sf.sspu.cn
}

\begin{abstract}
The multiresponse polynomial regression models are deal with by a linear combination to the responses for getting a manageable single-response model. Based on this method, prove that the symmetric design is optimal in the case of multiresponse polynomial regression models in symmetric domains.
\end{abstract}

Keywords-optimal designs; multiresponse polynomial regression models; symmetric design

\section{INTRODUCTION}

The polynomial regression model is widely used. It has been proved that the symmetric design is optimal in the case of singleresponse polynomial regression models in symmetric domains. But the construction of optimal designs for the multiresponse models is difficultly. However, the multiresponse polynomial regression models can be dealt with by a linear combination to the responses for getting a singleresponse model. Based on this method, it can be proved that the symmetric design is also optimal in the case of multiresponse polynomial regression models.

\section{MODEL TRANSFORMATION}

Consider the following linear models with $r$ responses

$$
E y_{i}(x)=f_{i}^{\prime}(x) \beta_{i}, \quad i=1,2, \cdots r,
$$

where $y_{i}$ is an observation on the ith responses, $f_{i}$ is a $k_{i} \times 1$ vector of the regression functions, $\beta_{i}$ is a $k_{i} \times 1$ vector of unknown constant parameters. A multivariate formulation of the models given in (1) is

$$
E y(x)=f^{\prime}(x) \beta \text {, }
$$

where $y=\left(y_{1}, \cdots, y_{r}\right)$ is the $r$-dimensional vector from the multivariate normal populations with the mean $f^{\prime}(x) \beta$ and a variance-covariance matrix $\Sigma=\left(\sigma_{i j}\right)_{r \times r}$, $f^{\prime}=\left(f_{1}^{\prime}, \cdots, f_{2}^{\prime}\right)$ is the $k$-dimensional vector and $k=\sum_{i=1}^{r} k_{i}, \beta=\operatorname{diag}\left(\beta_{1}, \cdots, \beta_{r}\right)$ is the block diagonal matrix of the parameters.

Let $c=\left(c_{1}, \cdots, c_{r}\right)^{\prime}$ be an arbitrary nonzero $r \times 1$ vector. From (2) we obtain the single-response model

$$
E y_{c}(x)=f^{\prime}(x) \beta_{c}=\sum_{i=1}^{r} c_{i} f_{i}^{\prime}(x) \beta_{i}
$$

where $y_{c}=y c=\sum_{i=1}^{r} c_{i} y_{i}, \beta_{c}=\beta c$

$=\left(c_{1} \beta_{1}^{\prime}, \cdots, c_{r} \beta_{r}^{\prime}\right)^{\prime}$ is a $k \times 1$ vector of parameters. Note that $y_{c}$, being a linear combination of normally distributed random variable, has the normal distribution with a variance

$$
\begin{aligned}
\sigma_{c}^{2} & =\operatorname{var}\left(y_{c}\right)=\operatorname{var}(y c)=c^{\prime} \Sigma_{c} \\
& =\sum_{i=1}^{r} c_{i}^{2} \sigma_{i i}+2 \sum_{i \neq j} c_{i j} c_{j i} \sigma_{i j}
\end{aligned} .
$$

The combination coefficients vector $C$ can be determined according to criteria. For example, Khuri(1985) developed a multivariate test for lack of fit test and let $C^{*}$ be the eigenvector of $G_{2}{ }^{-1} G_{1}$ associated with its largest eigenvalue. But in this paper, it does not change the result that $C$ is an arbitrary nonzero $r \times 1$ vector.

\section{SYMMETRIC POLYNOMIAL DESIGNS}

Consider the multiresponse polynomial regression models

$$
\begin{aligned}
E y_{i}(x) & =f_{i}^{\prime}(x) \beta_{i} \\
& =\beta_{i 0}+\beta_{i 1} x+\beta_{i 2} x^{2}+\cdots+\beta_{i k} x^{k} \\
i & =1,2, \cdots r,
\end{aligned}
$$

where $f_{i}(x)=\left(1, x, x^{2}, \cdots, x^{k}\right)^{\prime}$ is a $(k+1) \times 1$ vector of the regression functions, $\beta_{i}=\left(\beta_{i 0}, \beta_{i 1}, \cdots, \beta_{i k}\right)^{\prime}$ is a $(k+1) \times 1$ vector of unknown constant parameters.

Let $c=\left(c_{1}, \cdots, c_{r}\right)^{\prime}$ be an arbitrary nonzero $r \times 1$ vector. From (4) we obtain the single-response polynomial regression model

$$
\begin{aligned}
E y_{c}(x) & =f^{\prime}(x) \beta \\
& =\sum_{i=1}^{r} c_{i} f_{i}^{\prime}(x) \beta_{i}=\sum_{j=0}^{k} \sum_{i=1}^{r} c_{i} \beta_{i j} x^{j},
\end{aligned}
$$


where $f(x)=\left(1, x, x^{2}, \cdots, x^{k}\right)^{\prime}$ is a $(k+1) \times 1$ vector of the regression functions, $\beta=\left(\sum_{i=1}^{r} c_{i} \beta_{i 0}, \sum_{i=1}^{r} c_{i} \beta_{i 1}, \cdots, \sum_{i=1}^{r} c_{i} \beta_{i k}\right)^{\prime}$ is a $(k+1) \times 1$ vector of unknown constant parameters.

For the model (5), the experimental conditions $x_{1}, x_{2}, \cdots, x_{n}$ are assumed to lie in $[-1,1]$ and the experiment design is $\xi=\left(\begin{array}{llll}x_{1} & x_{2} & \cdots & x_{n} \\ p_{1} & p_{2} & \cdots & p_{n}\end{array}\right)$, where $p_{i}=\frac{N_{i}}{N}, \sum_{i=1}^{n} p_{i}=1 . N=\sum_{i=1}^{n} N_{i}$ is the total number of observations, and the information matrix

$$
M(\xi)=\int_{[-1,1]} f(x) f^{\prime}(x) d \xi=\sum_{i=1}^{n} p_{i} f\left(x_{i}\right) f^{\prime}\left(x_{i}\right) .
$$

An information matrix $M_{1}$ is at least as good as another information matrix $M_{2}$, relative to the criterion $\phi$, when $\phi\left(M_{1}\right) \geq \phi\left(M_{2}\right)$. It is essential that a reasonable criterion conforms:

$$
M_{1} \geq M_{2} \geq 0 \Rightarrow \phi\left(M_{1}\right) \geq \phi\left(M_{2}\right),
$$

$\phi\left(M_{1}+M_{2}\right) \geq \phi\left(M_{1}\right)+\phi\left(M_{2}\right)$, for all nonnegative definite $M_{1}$ and $M_{2}$.

The most prominent optimality criteria are the matrix means $\phi_{t}$, for $t \in(-\infty, 2]$, which enjoy many desired properties. The classical $A-, D-$ and $E$ - optimality criteria are special cases of $\phi_{t}$. Let $\lambda_{1}, \lambda_{2}, \cdots, \lambda_{p}$ denote the eigenvalues of a positive definite information matrix $M$. Then the matrix means $\phi_{t}$ is defined by $\phi_{t}=\left(\frac{1}{p} \sum_{i=1}^{p} \lambda_{i}^{t}\right)^{\frac{1}{t}}$. Because of the formula $V[\widehat{\beta}(\xi)]=M(\xi)^{-1}$, the matrix mean criteria can be expressed in terms of dispersion matrices as well. Criterion $\phi_{-1}$ is the $A-$ optimality criteria. Maximizing $\phi_{-1}(M)$ is equivalent to minimizing the trace of the corresponding dispersion matrix. The determinant criterion ( $D-$ optimality criteria) $\phi_{0}(M)$ is equal to $|M|$, and hence it induces the same preordering among information matrices as $|M|$. The extreme member of $\phi_{t}$ for $t \rightarrow-\infty$ yields the smallest-eigenvalue criterion ( $E-$ optimality criteria) $\phi_{-\infty}(M)=\lambda_{\min }(M)$.
According to the well known de la Garza phenomenon, let $\xi_{n}=\left(\begin{array}{cccc}x_{1} & x_{2} & \cdots & x_{n} \\ p_{1} & p_{2} & \cdots & p_{n}\end{array}\right)$ with $n>k+1$ be an $n$ point design for the LSE of $\beta$ in the polynomial model (5) of degree $k$. Then there exists a $(k+1)$-point $\operatorname{design} \xi_{k+1}^{*}=\left(\begin{array}{cccc}x_{1}^{*} & x_{2}^{*} & \cdots & x_{k+1}^{*} \\ p_{1}^{*} & p_{2}^{*} & \cdots & p_{k+1}^{*}\end{array}\right)$ for the LSE of $\beta$ in model (5) such that $M\left(\xi_{k+1}^{*}\right)=M\left(\xi_{n}\right)$.

First we consider the reflection operation. Let $\xi$ be a design for the LSE of $\beta$ on $\chi=[-1,1]$ in the polynomial model (5). The reflected design $\xi^{R}$ is given by $\xi^{R}=\left(\begin{array}{cccc}-x_{1} & -x_{2} & \cdots & -x_{n} \\ p_{1} & p_{2} & \cdots & p_{n}\end{array}\right)$, then
$M\left(\xi^{R}\right)=Q M(\xi) Q$,

where $Q=\operatorname{Diag}(1,-1,1,-1, \cdots, \pm 1)$.

The symmetrised design

$$
\begin{aligned}
\bar{\xi} & =\frac{1}{2}\left(\xi+\xi^{R}\right) \\
& \equiv\left(\begin{array}{ccccccc}
x_{1} & -x_{1} & x_{2} & -x_{2} & \cdots & x_{n} & -x_{n} \\
\frac{p_{1}}{2} & \frac{p_{1}}{2} & \frac{p_{2}}{2} & \frac{p_{2}}{2} & \cdots & \frac{p_{n}}{2} & \frac{p_{n}}{2}
\end{array}\right),
\end{aligned}
$$

Assigns the weight $\frac{p_{i}}{2}$ to $x_{i}$ and $-x_{i}$ for each $i$. The information matrix of $\bar{\xi}$ is

$$
M(\bar{\xi})=\frac{1}{2}(M(\xi)+Q M(\xi) Q),
$$

where all odd moments are zero and the even moments are equal to the corresponding moments of the original design $\xi$. Hence the averaging operation simplifies information matrices by letting all odd moments vanish. Since $M\left(\xi^{R}\right)$ is obtained from $M(\xi)$ by the similarity transformation (6), $M\left(\xi^{R}\right)$ and $M(\xi)$ have the same eigenvalues.

From the above, it follows that any optimality criterion which is a function of the eigenvalues of the information matrices will be invariant with respect to the reflection operation and superadditive of $\phi$ (with respect to the reflection) imply 


$$
\begin{aligned}
\phi[M(\bar{\xi})] & =\phi\left\{\frac{1}{2}\left[M(\xi)+M\left(\xi^{R}\right)\right]\right\} \\
& \geq \frac{1}{2}\left\{\phi[M(\xi)]+\phi\left[M\left(\xi^{R}\right)\right]\right\} \\
& =\phi[M(\xi)] .
\end{aligned}
$$

Thus symmetrization improves the value of the criterion $\phi$, or at least maintains the same value, provided that $\phi$ is superadditive and invariant with respect to the reflection. Therefore, for such criteria, we may confine ourselves to the class of symmetric designs.

\section{SYMMETRIC DESIGNS FOR QUADRATIC REGRESSION}

Let $\xi_{3}$ be a symmetric 3 -point design on $\chi=[-1,1]$ for the LSE of $\beta=\left(\sum_{i=1}^{r} c_{i} \beta_{i 0}, \sum_{i=1}^{r} c_{i} \beta_{i 1}, \sum_{i=1}^{r} c_{i} \beta_{i 2}\right)^{\prime}$ in the quadratic model

$$
E Y_{c}=\sum_{i=1}^{r} c_{i} \beta_{i 0}+\sum_{i=1}^{r} c_{i} \beta_{i 1} x+\sum_{i=1}^{r} c_{i} \beta_{i 2} x^{2}
$$

According to the E.P. Liski(2002) result, there exists a symmetric 3 -point design $\xi_{3}^{*}=\left(\begin{array}{ccc}-1 & 0 & 1 \\ \frac{p}{2} & 1-p & \frac{p}{2}\end{array}\right)$ with $p<1$ such that $M\left(\xi_{3}^{*}\right) \geq M\left(\xi_{3}\right)$.

The characteristic function of $M\left(\xi_{3}^{*}\right)$ is

$$
F(\lambda)=(p-\lambda)[(1-\lambda)(p-\lambda)-p],
$$

which yields the eigenvalues of $M\left(\xi_{3}^{*}\right)$ :

$$
\begin{aligned}
& \lambda_{1}=\frac{1}{2}(p+1)+\frac{1}{2} \sqrt{5 p^{2}-2 p+1}, \\
& \lambda_{2}=p \\
& \lambda_{3}=\frac{1}{2}(p+1)-\frac{1}{2} \sqrt{5 p^{2}-2 p+1} .
\end{aligned}
$$

The optimal designs are as follows:

A-optimal design: $\left(\begin{array}{ccc}-1 & 0 & 1 \\ \frac{1}{4} & \frac{1}{2} & \frac{1}{4}\end{array}\right)$,
$D$ - optimal design:
$\left.\begin{array}{cccc}-1 & 0 & 1 \\ \frac{1}{3} & \frac{1}{3} & \frac{1}{3}\end{array}\right)$,
$E$ - optimal design:
$\left.\begin{array}{ccc}-1 & 0 & 1 \\ \frac{1}{5} & \frac{3}{5} & \frac{1}{5}\end{array}\right)$.

\section{REFERENCES}

[1] E.P. Liski, N.K.Mandal, K.R.Shah, and B.K.Sinha, Topics in Optimal Design. Springer Press, 2002.

[2] A.I. Khuri, “A test for lack of fit of a linear multiresponse model," Technometrics., vol. 27, pp. 213-211, 1985.

[3] A.C. Atkinson, and A.N.Donev, Optimal experimental design. Oxford: Oxford University Press, 1992.

[4] V.V. Fedorov, Theory of optimal Experiments. ACADEMIC Press, 1972.

[5] A. de la Garza, "Spacing of information in polynomail regression," Annals of Mathematical Statistics, vol. 25, pp. 123-130, 1954.

[6] P. Whittle, "Some general points in the theory of optimal experimental desig,” Journal of the Royal Statistical Society. Series B, vol. 35 pp. 123-130, 1973. 